\title{
Male Breast Carcinoma; Indepth Presentation Viz A Viz Female Breast
}

\author{
Authors \\ Dr Pragya Garg, Dr Supreethi Kohli \\ E.S.I.Post Graduate Institute of Medical Science and Research, Basai Darapur, New Delhi, Delhi 110015, \\ India \\ Corresponding Author \\ Dr Supreethi Kohli
}

Tower 5, Flat Number: 1403, Sushant Estate, Sector 52,Gurgaon, Haryana Pin Code: 122002

Email: suprkohli@gmail.com

\begin{abstract}
Male breast cancer is exceptionally rare and accounts for less than $0.25 \%$ of male malignancies and approximately 0.5-1\% of all breast cancer (both genders). Our article emphasizes the pathological and radiological differences of male breast cancers from female breast cancers. Mammography of the male breast, constitutes less than 1\% of all mammograms performed, commonest lesion imaged being gynecomastia. So, there is less familiarity with the imaging appearances of male breast cancers. We report a case of invasive ductal carcinoma in a 62 years male and provide a comparison with presentations and manifestations in female. Key words: Breast cancer, Mammography, Ultrasonography, Male, Female.
\end{abstract}

\section{Introduction}

We present a case of concurrent gynecomastia and breast cancer. Gynecomastia is the most common cause of breast enlargement. Gynecomastia, clinically presents as enlarged, soft, tender breast. A mass may be palpable in the retro-areolar region. Patients with breast malignancy complains of sudden and rapid breast enlargement and tenderness of lump. Differentiation between benign and malignant masses is critical. On the basis of combination of morphology and distribution, mammography allows differentiation between benign and malignant breast disease in male patients with a high sensitivity (92\%) and specificity $(90 \%)^{[1] .}$

\section{Case Report}

A 62 year well built male, presented with complaints of pain and swelling in the left retroareolar region. The right breast was also enlarged. The patient denied any family history of breast cancer and had no history of trauma to the breast. He was not any medication. On examination; left nipple and areola was retracted. Overlying skin was oedematous and inflamed. An oozing superficial ulcer was seen at 5 o'clock position. (figure1) The patient underwent a bilateral digital diagnostic mammogram with mediolateral oblique (MLO) and craniocaudal (CC) views of both breasts (Hologic Selenia). Left breast showed evidence of a poorly marginated, irregular, hyperdense lesion with spiculated margins in retroareolar region. Nipple 
was retracted with associated thickening of overlying skin and subcutaneous tissue. Ipsilateral Pectoralis appears normal. The lesion was not associated with any calcification. Lymph node with imperceptible fatty hilum is seen in left axilla.No evidence of any intramammary lymphadenopathy was seen(figure2,figure3). Right breast showed benign diffuse proliferation of normal fatty tissue suggestive of pseudogynecomastia. Targeted ultrasonography of left breast was performed utilizing an $11 \mathrm{MHz}$ transducer (Philips IE33). The retroareolar density in the left breast seen on mammogram corresponded to an irregular, poorly marginated, hypoechoic lesion with angulated margins and also showed posterior acoustic enhancement (figure 4). No e/o any regional calcification was seen. Normal left axillary lymph nodes were present. The patient subsequently underwent a core biopsy from the lesion. Pathology results showed invasive carcinoma. Estrogen and progesterone receptors were negative. The patient was treated with left total mastectomy and sentinel lymph node biopsy, which was positive. Surgical pathology showed invasive breast carcinoma of no special type (NST).

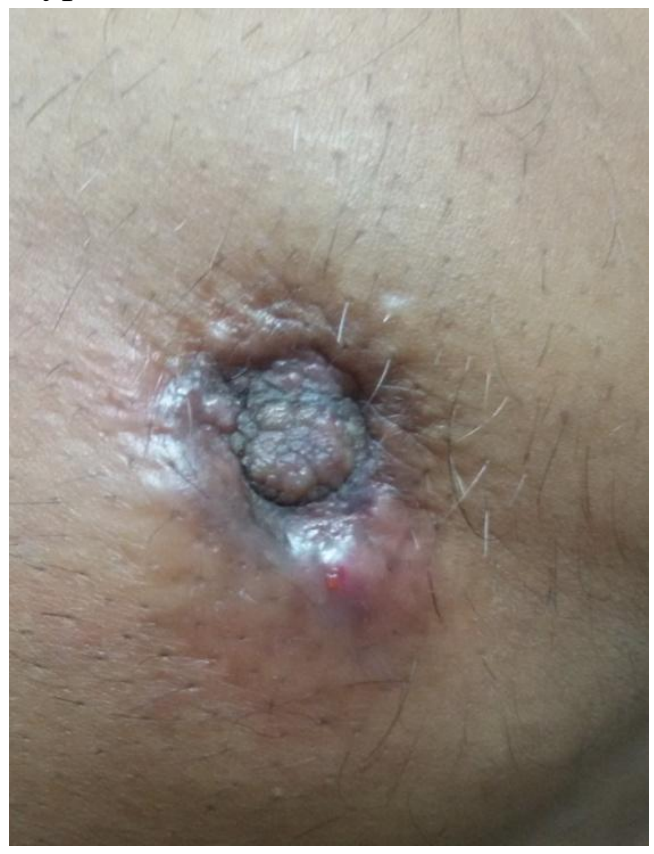

FIGURE 1:Enlarged left breast with retracted left nipple and areola with oedematous and inflamed overlying skin and subcutaneous tissue. An oozing superficial ulcer was seen at 5 o'clock position

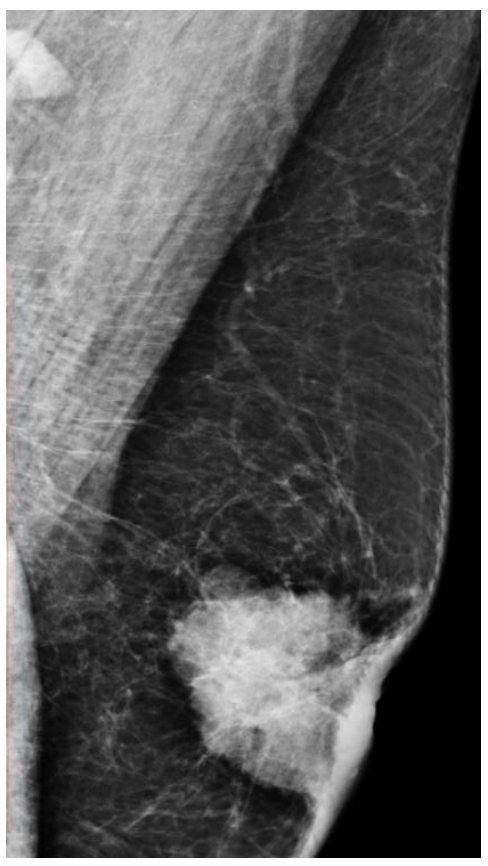

FIGURE 2: Digital diagnostic mammogram with mediolateral oblique (MLO. Left breast showed evidence of a spiculated, hyperdense lesion in retroareolar region. Nipple was retracted with associated thickening of overlying skin and subcutaneous tissue. Ipsilateral Pectoralis appears normal. The lesion was not associated with any calcification. Axillary lymph node with imperceptible fatty hilum seen.No intramammary lymphadenopathy was seen

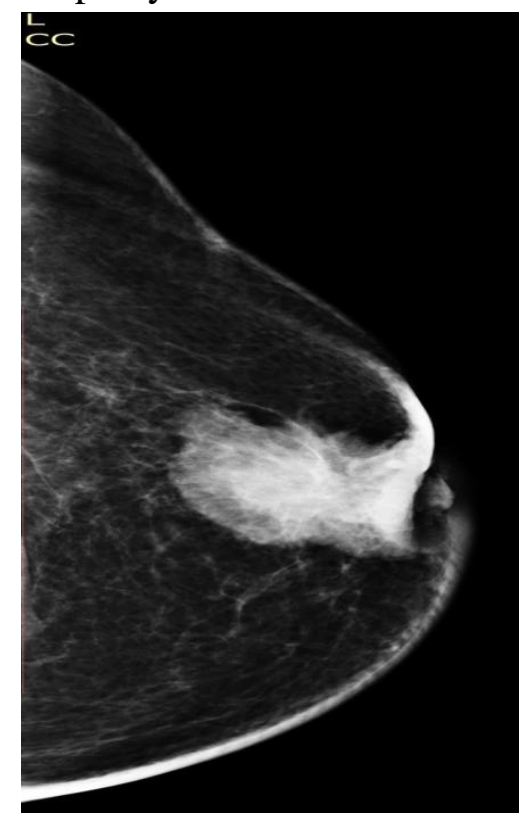

FIGURE 3: Digital diagnostic mammogram craniocaudal (CC) view of left breast. (Hologic Selenia). Left breast showed evidence of a poorly 
marginated, irregular, hyperdense lesion with spiculated margins in retroareolar region. Nipple was retracted with associated thickening of overlying skin and subcutaneous tissue. No intramammary lymphadenopathy seen

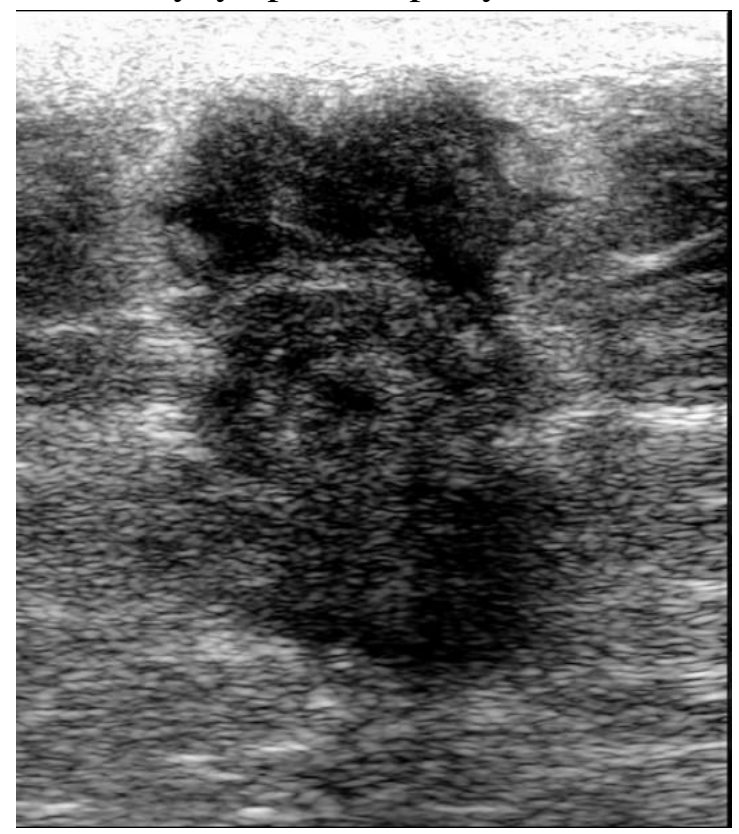

FIGURE 4: Targeted ultrasonography of left breast was performed utilizing an $11 \mathrm{MHz}$ transducer (Philips IE33). The retroareolar region of left breast,shows an irregular, poorly marginated, hypoechoic lesion with angulated margins and also showed posterior acoustic enhancement. No e/o any regional calcification was seen

\section{Discussion}

Normal adult male breasts are composed of skin, subcutaneous fat, atrophic ducts and stromal elements. Preponderance of skin and fat elements accounts for the typical mammographic appearance of the normal male breast. Cooper ligaments are absent in male breasts. At birth, the male and female breasts are the same. Histologically, the normal male breast contains subareolar ducts similar to those found in prepubertal girls. In most males these do not develop further until stimulated by variety of drugs or hormones. One of the prominent differences between gynecomastia and the female breast is that lobule formation is extremely rare. Therefore, breast conditions related to lobular proliferation, such as fibroadenoma, phyllodes tumor, invasive lobular carcinoma, and lobular carcinoma in situ, are extremely uncommon in men. Conditions related to ductal and stromal proliferation, such as gynecomastia, invasive ductal carcinoma, ductal carcinoma in situ, and papillary neoplasm, may occur in men ${ }^{(2)}$. The major difference between the cancers in women and men is also because of the site of origin. Cancers in women originate primarily, within the terminal ductul lobular units peripherally and only secondarily involve the central ducts. Whereas, in men breast tissue is subareolar. Yap et al. ${ }^{(3)}$ in their study, reported that $88 \%$ of breast cancers in their study were subareolar with nipple involvement.

Carcinoma of the male breast is an unusual lesion with a frequency equalling only about $0.9 \%$ of the occurrence of female breast cancer ${ }^{(3)}$. This low incidence in men justifies the fact that mammography in males cannot be used as a screening modality and can only be used for diagnostic purposes. The peak incidence of breast carcinomas in males is in the fifth and sixth decades. However, a case of a 6-year-old boy with breast cancer has been reported. Thymic irradiation was given to the child at the time of birth ${ }^{(4)}$. In men, breast cancer is typically diagnosed at an age approximately 5-10 years older than their female counterparts. In addition, men usually present at a more advanced stage of cancer than do women owing to a delay in diagnosis.

Factors predisposing a male to breast carcinoma are; elevated estrogen levels, hormone therapy for prostate carcinoma, gynecomastia, Klinefelter's syndrome ${ }^{(5,6)}$ family history, a history of chest irradiation and BRCA1 or BRCA2 mutation. Breast cancer_in men_manifests clinically as a hard, fixed, painless lump that are usually centrally located and may be fixed to the skin or to the underlying Pectoralis muscle. Bloody nipple discharge, may be associated with nipple ulceration, as has been reported in up to $25 \%$ of cases ${ }^{(7)}$. Ipsilateral axillary lymphadenopathy at 
presentation is commoner in men_with breast cancer $^{(8)}$.

Breast cancer in men is almost always clinically evident and therefore often not evaluated with mammography ${ }^{(9,10)}$. Malignant masses show variable appearances; they may be well marginated or may have a stellate appearance like that of scirrhous carcinoma in the female. Well marginated /Circumscribed masses should be regarded with suspicion, as they can represent carcinoma in men ${ }^{(11,12)}$. As well defined breast nodules in men are likely to represent cancer, biopsy of these lesions is indicated ${ }^{(13)}$. In a study of 57 patients ${ }^{(14)}$ describing the imaging (mammography_and sonography) features of primary breast cancer_in men._Calcifications were found in $31 \%$ of male breast cancer patients . Forty-seven percent of calcifications were pleomorphic and $18 \%$ were punctuate. Study by Dershaw et al, ${ }^{(15)}$ showed calcifications in $13 \%$ of patients. Through both these studies, it was found that calcifications which are generally considered benign in women may be associated with malignancy in men. Male breast cancers have similar Sonographic features as in females except for Papillary carcinomas, which presents as a prominent cystic component in males as compared to females ${ }^{(16,17)}$.

The most common histologic type of breast cancer in men_is infiltrating ductal carcinoma, accounting for $80-85 \%$ of cancer cases in men ${ }^{(18)}$. Ductal carcinoma in situ is the second most common subtype accounting for about $5 \%$ of all cases ${ }^{(14)}$. Other less common subtypes include infiltrating mammary carcinoma with mixed features and invasive papillary carcinoma ${ }^{(14)}$. Comedo, tubular, lobular, medullary, and mucinous in situ lesions are absent or less common among_men_than women, whereas in situ papillary lesions were relatively more common among_men_than women ${ }^{(19)}$. Lobular cancers are less common in men than in women because lobule formation is rare in men, even in those with gynecomastia ${ }^{(20)}$. Only $1.5 \%$ of breast cancers_in men_are lobular, as opposed to $12 \%$ of breast cancers in women ${ }^{(20)}$. Men with breast cancers carry a poorer prognosis, as and when compared with that of females. This can be partly due to detection at an advanced stage and lack of proper awareness. The prognosis for breast cancer depends on lymph node status, tumor size, and duration of symptoms. In a study by Keith et al ${ }^{(16)}$ on 97 breast cancer patients, none of the patients with intraductal or intracystic carcinoma died of cancer. Survival of the entire group of men with invasive carcinoma was $40 \%$ after ten years. The ten-year survival for men with negative nodes was $79 \%$, for men with positive nodes $11 \%$. Comparison was done with similar aged females and it was found that men had a significantly lower survival rate. This poorer prognosis was limited to those men with pathologically positive axillary nodes. No reports of mammography in post mastectomy men are available.

\section{Conclusion}

Carcinomas in the male breast, occurs with $<1 \%$ frequency as compared with that of female breast cancer. Mammography in males is used as a diagnostic modality in contrast to, being used as a screening modality in females. Cancers in women originate primarily, within the terminal ductul lobular units and are peripheral in location. Whereas, in men breast tissue is subareolar. So, majority of carcinomas are subareolar in location. Breast cancers are typically diagnosed at an age approximately 5-10 years older than females and unfortunately, at a more advanced stage. Bloody nipple discharge, associated nipple ulceration and Ipsilateral axillary lymphadenopathy are commoner at presentation in men. Well marginated/Circumscribed lesions should be regarded with suspicion in males. Sonographic features are similar to those seen in females except for Papillary carcinomas, which presents as a prominent cystic component in males. The prognosis for breast cancer depends on lymph node status, tumour size, and duration of symptoms. On comparison with similar aged females, it was found that men had a significantly lower survival rate. 


\section{References}

1. Evans GF, Anthony $\mathrm{T}$ et al. The diagnostic accuracy of mammography in the evaluation of male breast disease. Am J Surg 2001;181(2): 96-100.

2. Kopans DB. Breast imaging. Philadelphia, Pa: Lippincott Williams \& Wilkins, 2007

3. Yap H, Tashima Cet al. Male breast cancer, a natural history study. Cancer 1979;44:748-754.

4. Simpson J, Barson A. Breast tumors in infants and children: a 40-year review of cases at a children's hospital. Can Med Assoc J 1969;1Oi :100-i 02.

5. A. P. Hittmair, R. A. Lininger et al. Ductal carcinoma in situ (DCIS) in the male breast.Cancer 1998; vol. 83, pp. 21392149.

6. J. Armstrong, C. Saunders et al. Male breast ductal carcinoma in situ. ANZ Journal of Surgery 2003; 73: 774-775.

7. Sandler B, Carman C et al. Cancer of the male breast. Am Surg 1994; 60:816-820.

8. Joshi MG, Lee AKC et al. Male breast carcinoma: an evaluation of prognostic factors contributing to poorer outcome. Cancer 1996; 77:490-498.

9. Kalisher U, Payster AG. Xerographic manifestations of male breast. AJR 1975; $125: 656-661$.

10. Dershaw DD. Male mammography. AJR1986; 146:127-131.

11. Stavros TA, Rapp CL, Parker SH. Breast ultrasound. Philadelphia, PA: Lippincott Williams \& Wilkins, 2004:712-739 .

12. Michels UG, Gold RHet al. Radiography of gynecomastia and other disorders of the male breast. Radiology 1977 ; 122:117122.

13. Onimet-Oliva D, Hebert $G$ et al. Radiographic characteristics of male breast cancer. Radiology 1978; i29:37-40 .
14. Jean Mathew, George H. Perkins et al. Primary Breast Cancer in Men: Clinical, Imaging, and Pathologic Findings in 57 Patients. AJR 2008;191 (6):1631-1639.

15. Dershaw DD, Borgen Pi et al. Mammographic findings in men with breast cancer. AJR 1993; 160: 267-270.

16. Heller KS, Rosen PP et al. Male breast cancer: a clinicopathologic study of 97 cases. Ann Surg 1978; 188 :60-65.

17. Visfeldt J, Sheike O. Male breast cancer. 1. Histologic typing and grading of 187 Danish cases. Cancer 1973; 32:985-990.

18. Jellici E, Malago R et al. Imaging of the male breast. Radiol Med 2005; 110:574588.

19. Anderson WF, Devesa SS. In situ male breast carcinoma in the Surveillance, Epidemiology, and End Results database of the National Cancer Institute. Cancer 2005; 104:1733-1741.

20. Giordano SH. A review of the diagnosis and management of male breast cancer. Oncologist 2005; 10:471-479 